\title{
Dependency of continuum model parameters on the spatially correlated pore structure studied by pore-network drying simulations
}

\section{Lu, X.; Kharaghani, A.*; Tsotsas, E.}

Thermal Process Engineering, Otto von Guericke University Magdeburg, Magdeburg, Germany *E-mail of the corresponding author: abdolreza.kharaghani@ovgu.de

\begin{abstract}
Pore-network simulations are carried out for monomodal and bimodal pore structures with spatially correlated pore-size distributions. The internal and surface relationships between the partial vapor pressure and saturation as well as the moisture transport coefficient for these model porous structures are identified from the post-processing of the corresponding pore-network model solutions. The simulation results show that the deviation of the partial vapor pressure from the saturation vapor pressure in the presence of liquidwhich is referred to as non-local equilibrium effect - in the bimodal pore structures is less pronounced than in the monomodal pore structures. For the monomodal pore structures the moisture transport coefficient profile is not unique over the entire drying process, whereas this profile depends marginally on the drying history of the bimodal pore structures. Finally the ability of the continuum model to predict the results of the pore-network simulations for multiple realizations of the pore space is assessed.
\end{abstract}

Keywords: Scale transition, Moisture transport coefficient, Pore structure, Discrete model, Continuum model 
Dependence of continuum model parameters on the spatially correlated pore structure studied by pore-network drying simulations

\section{Introduction}

The drying characteristics of porous media can essentially be described via modeling on two different length scales: at the effective-medium scale, continuum models (CM) based on volume averaging have been developed, in which the underlying transport phenomena are described by gradients in spatially averaged quantities and controlled by nonlinear effective parameters [1]. While the CMs are readily competent on the application side, they require macroscopic parameters and suffer from major shortcomings in theoretical aspects. The alternative approach operates at the pore scale and is referred to as discrete porenetwork modeling. Discrete network models with different representations of the pore space have been developed, which can be divided into two categories: In the work of Yiotis et al. [2] the entire pore space is represented by pore bodies, leaving the throats as conductors and capillary barriers. This representation is suitable for the large pore volume. It may however fail to represent properly the structures with small pores. Prat et al. [3] and Metzger et. al. [4] approximated the void space with hydraulic pore throats and numerical pore nodes. This model is referred to as throat-node-model (TNM) in this work.

Metzger et al. [4] investigated how important is the role of pore structure (with monomodal pore size distribution (PSD) and bimodal PSD) on the intraparticle heat and mass transfer and thus drying kinetics. Vey recently, Moghaddam et al. [5] assessed the classical continuum model by the help of pore network simulations. In these simulations pore networks were generated with a throat radius distribution of $250 \pm 25 \mu \mathrm{m}$, resulting in a network porosity of 0.594 . In these networks the pore space volume is overestimated due to the throat overlaps at nodes. This void space overestimation is avoided in throat-pore-model (TPM), which is developed in this work. Moghaddam et al. [5] calculated the continuum model parameters from TNM simulations. The results indicated that the moisture transport coefficient and the vapor pressure-saturation relationship are not unique over the entire drying process.

The relationships between the pore structure and the CM parameters are unknown. The aim of this work is to study the dependence of the parameter functions - the internal and surface relationships between the partial vapor pressure and saturation as well as the moisture transport coefficient - on the spatially correlated structures by means of the TPM. The ability of the continuum model to predict the results of the TPM simulations for multiple realizations of the pore space is also assessed.

\section{Model descriptions}

\subsection{The continuum model}

Assuming isothermal condition and constant gas pressure during the entire drying process, the mass transport is dominated in the direction perpendicular to the medium surface which 
provides the evaporation demand. According to the total conservation of moisture (liquid and vapor phases), the continuum model is thus expressed by:

$$
\varepsilon \frac{\partial S}{\partial t}=\frac{\partial}{\partial z}\left(D(S) \frac{\partial S}{\partial z}\right),
$$

with the boundary condition of no accumulation at the interface:

$$
\dot{m}_{v}=-\frac{D_{v a} \tilde{M}_{v}}{\widetilde{R} T \delta} \ln \left(\frac{P-P_{v \infty}}{P-\varphi P_{v}^{*}}\right),
$$

where $\varepsilon, S$, and $t$ (s) denote the porosity, the local saturation $(0<S \leq 1)$ and the time, respectively; $P_{v \infty}(\mathrm{Pa})$ the vapor pressure in the bulk air, $P(\mathrm{~Pa})$ the atmosphere pressure, $\delta$ (m) the thickness of the diffusive mass boundary layer, which is located between the network open surface and the bulk air. The time-dependent moisture transport coefficient $D$ $\left(\mathrm{m}^{2} / \mathrm{s}\right)$ as well as the boundary conditions need to be known in order to solve Eq. 1. Two parameters $D$ and $\varphi$, which is called the vapor pressure-saturation, are estimated from pore network simulations.

\subsection{Pore network drying model}

The pore network model is defined on a three-dimensional regular lattice of pores and throats. The pores are spherical space and throats are cylindrical tubes (Figure 1a). The throat and pore radii are randomly set from normal distributions with corresponding means and standard deviations. The pore radii are chosen such that the pore radius is larger or equal with the largest radius of neighboring throats. Initially all throats and pores are saturated with liquid existing as free water. Periodical boundary conditions are applied on the lateral faces of the network. The extended boundary layer is considered in this model, which is a playground for the external mass transfer of vapor.

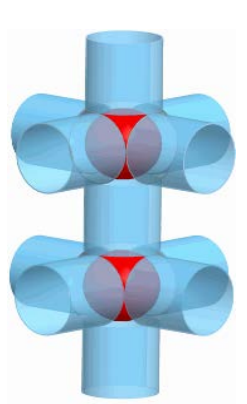

(a)

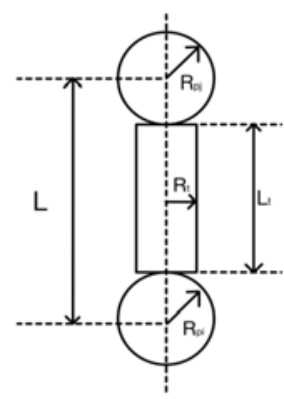

(b)

Figure 1 Schematics of throat-pore-model (TPM) in $3 D(a)$ and in $2 D(b)$. 
Three kinds of pores and three kinds of throats are classified in the algorithm: empty pores and throats; full pores and throats; and partially filled pores and throats. The pores contain variables of interests, namely the liquid pressure and the vapor partial pressure. Transport phenomena are described at the discrete level of pores and throats in one spatial dimension. In this model, the center distance between two pores ( $i$ and $j$ in Figure $1 \mathrm{~b}$ ) are fixed ( $L=1000 \mu \mathrm{m})$. The radii of pores $\left(R_{p}\right)$ and throats $\left(R_{t}\right)$ are drawn randomly from normal distributions. The throat length $\left(L_{t}\right)$ depends on the radii of two pores located at throat ends.

The Stefan law is used to describe the vapor transport in the gas-filled region of the network and the boundary layer. The quasi-steady vapor diffusion between two pores is described by:

$$
\sum_{j} \dot{M}_{v, i j}=\sum_{j} g_{v, i j} P \ln \left(\frac{P-P_{v}^{j}}{P-P_{v}^{i}}\right), g_{v, i j}=\frac{D_{v a} \tilde{M}_{v}}{\tilde{R} T\left(\frac{R_{p i}}{A_{p i}}+\frac{L_{t}}{A_{t}}+\frac{R_{p j}}{A_{p j}}\right)},
$$

where $A_{p}$, and $A_{t}\left(\mathrm{~m}^{2}\right)$ denote the cross section area of pore (i.e. $\pi R_{p}{ }^{2}$ ) and of throat (i.e. $\left.\pi R_{t}^{2}\right), D_{v a}\left(\mathrm{~m}^{2} / \mathrm{s}\right)$ the diffusion coefficient of vapor in air, $\tilde{R}(\mathrm{~J} / \mathrm{kmol} \mathrm{K})$ the universal gas constant, $\tilde{M}_{v}(\mathrm{~kg} / \mathrm{kmol})$ the molar mass of vapor and $T(\mathrm{~K})$ the temperature. $P_{v}$ is the vapor pressure at pores.

The Poiseuille's law is used to describe the liquid viscous flow as:

$$
\sum_{j} \dot{M}_{w, i j}=\sum_{j} g_{w, i j}\left(P_{w}^{i}-P_{w}^{j}\right), g_{w, i j}=\frac{\pi \rho_{1}}{8 \mu} \frac{1}{\left(\frac{R_{p i}}{R_{p i}{ }^{4}}+\frac{L_{t}}{R_{t}{ }^{4}}+\frac{R_{p j}}{R_{p j}{ }^{4}}\right)},
$$

where $\rho_{l}\left(\mathrm{~kg} / \mathrm{m}^{3}\right)$ denotes the liquid mass density, and $\mu\left(\mathrm{m}^{2} / \mathrm{s}\right)$ the liquid viscosity. Considering the viscous and capillary forces at the same time, stationary and moving menisci are determined in an iterative manner. The evaporation drives the drying process leading to drainage of moving menisci. The reimbibition can locally occur depending on the local liquid pressure. The time steps forward when one meniscus empties or (re)fills at every moment during drying. In the next steps the mass balance for vapor and liquid phases are solved again. Details on the algorithm can be found in Metzger et al. [4].

In this paper, we generated two pore network structures with monomodal PSD and bimodal PSD. The TPM was applied to generate these two structures. In the network with monomodal PSD, throat and pore radii are drawn randomly from a normal distribution with the given values of standard deviation and mean radius. The network with bimodal PSD is constructed with micro- and macro-channels. The macro-channels are placed vertically in the network. 


\subsection{Parameter functions}

The PN simulations yield the vapor and liquid pressure fields as well as the liquid distribution over time. This information is used to determine both the internal and surface relationships between vapor pressure and saturation $\varphi$ as well as the moisture transport coefficient $\mathrm{D}$. The finite volume method is applied to determine these parameter functions. A three-dimensional network is virtually divided into horizontal slices with constant thickness $\Delta \mathrm{z}$ at distinct times during the drying process. Details of this method is explained in Moghaddam et al. [5].

\section{Simulation results}

The continuum model parameters are determined from the simulations conducted for monomodal and bimodal pore structures with spatially correlated pore-size distributions. In order to reduce the level of uncertainty on parameter estimates, 10 realizations of pore network were considered. The structural characteristics of PN are given in Table 1.

Table 1 Structural characteristics of pore networks.

\begin{tabular}{lll}
\hline Structural property & Monomodal PSD & Bimodal PSD \\
\hline Network size (pore) & $25 \times 25 \times 51$ & $25 \times 25 \times 51$ \\
Boundary layer size (node) & $25 \times 25 \times 10$ & $25 \times 25 \times 10$ \\
Mean radius $(\mu \mathrm{m})$ & 250 & $\begin{array}{l}\text { micro-channel: } 200 \\
\text { macro-channel: } 350\end{array}$ \\
Standard deviation $(\mu \mathrm{m})$ & 25 & 25 \\
Center distance between two pores $(\mathrm{mm})$ & 1 & 1 \\
Network porosity & 0.35 & 0.337 \\
\hline
\end{tabular}

Figure 2 shows the calculated moisture transport coefficient for the two pore structures. As can be seen, the D-S curves depend strongly on the drying history in the networks with monomodal PSD. This dependency is however less pronounced in the networks with bimodal PSD. Similar moisture transport coefficient profiles are identified by Moghaddam et al. [5], but only for the pore structures with monomodal PSD. As can also be seen, the value of moisture transport coefficient increases when altering the pore structure from monomodal PSD to bimodal PSD, especially in the period during which moisture transport occurs mainly through the liquid phase $\left(S_{\text {net }}=1 \cdot 0-0.7\right)$. In the network with bimodal PSD the local saturation varies rather uniformly and confines within a small range of $S=1.0$ 0.65 due to the presence of macro-channels. However, in the network with monomodal PSD the dry region develops rather early due to the presence of strong viscous effect. 
Hence the saturation profiles vary with a broad range of $S=1.0-0.2$ during the first drying period $\left(S_{n e t}=1.0-0.7\right)$. In the work of Moghaddam et al. [5], conducted with the pore structures of monosized PSD, the range of local saturation variation is wider $S=1.0-0$ during the first drying period. One reason for this wide variation could be due to the overestimation of the throat volume in the TNM.

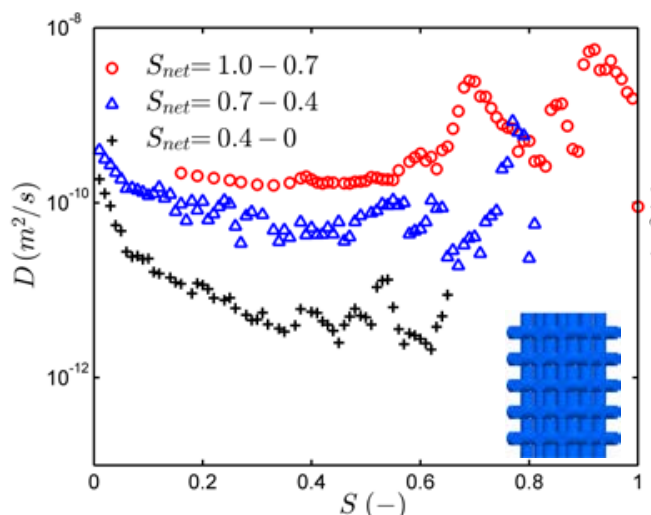

(a)

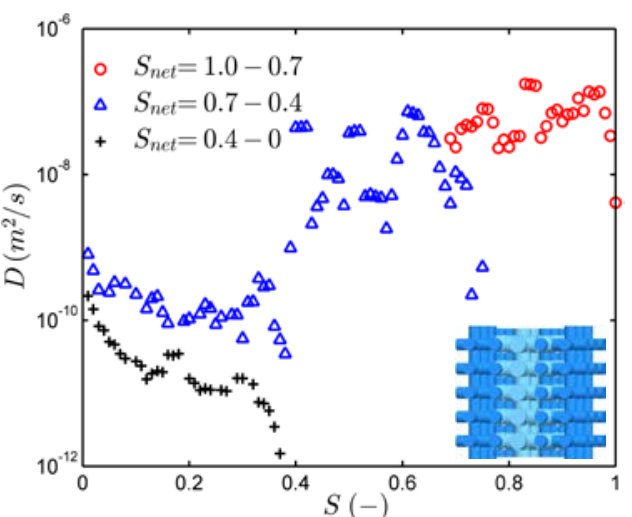

(b)

Figure 2 Simulated moisture transport coefficients $D$ for the pore structures with (a) monomodal PSD and (b) bimodal PSD. The values are averaged over 10 realizations for local saturation $S$ intervals.

The coefficient profiles are similar for the both pore structures when moisture transport occurs mainly through the vapor phase. This is so because the moisture transport in the vapor phase is driven by the vapor pressure difference, which is related to the liquid structure (or interfacial area).

The behavior of moisture transport coefficient in the vapor phase can be better understood from the vapor pressure-saturation relationship $(\varphi)$. Moghaddam et al. [5] observed a nonelocal equilibrium (NLE) effect, i.e. the deviation of the vapor pressure from the saturation vapor pressure in two-phase zone, in the pore network simulations. This effect is also observed in the both pore structures (Figure 5). The NLE is strong in the network with monomodal PSD. The liquid controlled range is enlarged in the networks with bimodal PSD due to an efficient capillary pumping. Figure 5 indicates that the vapor pressure deviates significantly from the saturation pressure at low saturation in the both pore structures. The NLE effect appears at early time of the drying process $\left(S_{n e t}=1.0-0.65\right)$ in the networks with monomodal PSD. This is so because of the drying front stabilization by viscous force. However, this effect appears later $\left(S_{n e t}=0.55-0.45\right)$ in the networks with bimodal PSD.

The two parameter functions calculated from the PN simulations are plugged into the continuum model (Eq. 1). The results obtained from the solution of the CM and from the 
PN simulations are shown together in Figure 6. For the network with monomodal PSD, the CM predicts fairly the saturation profiles, except those profiles at high network saturation where the moisture transport coefficient values are scattered. This discrepancy may nonetheless be reduced when the parameters are determined for more realizations of larger pore networks.

A fairly good agreement between the CM predictions and the discrete simulations of the pore structures with bimodal PSD is observed (see Fig. 6b). The fact that at high saturation the variation of the moisture transport coefficient value is limited to a small range has led to this good match.

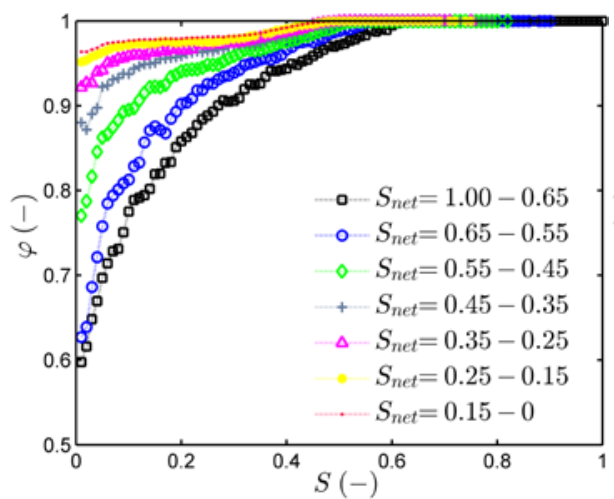

(a)

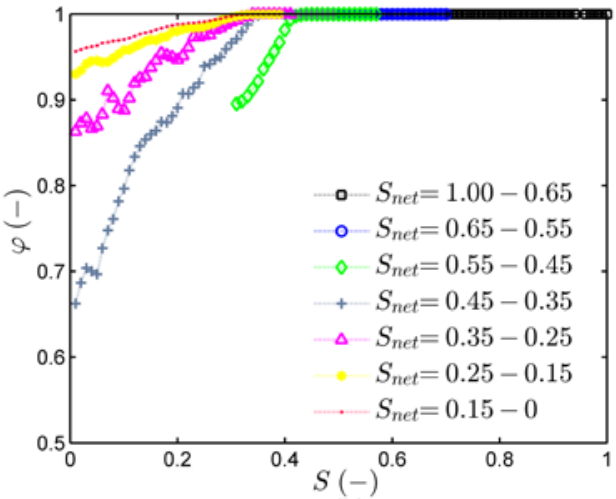

(b)

Figure 5 The vapor pressure-saturation relationships for the pore structures with (a) monomodal PSD and (b) bimodal PSD. The values are averaged over 10 realizations.

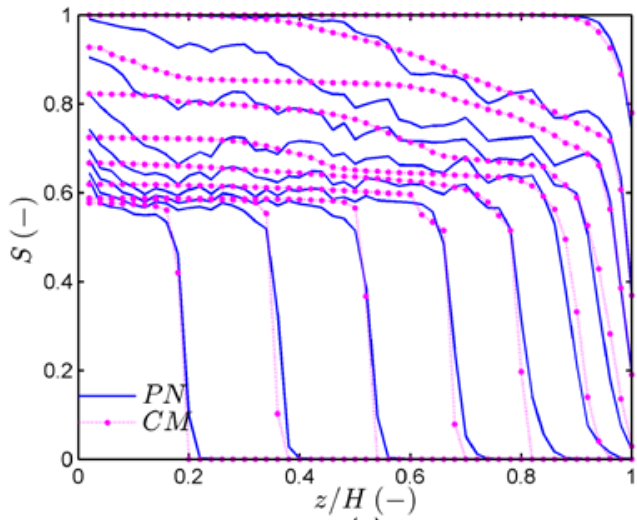

(a)

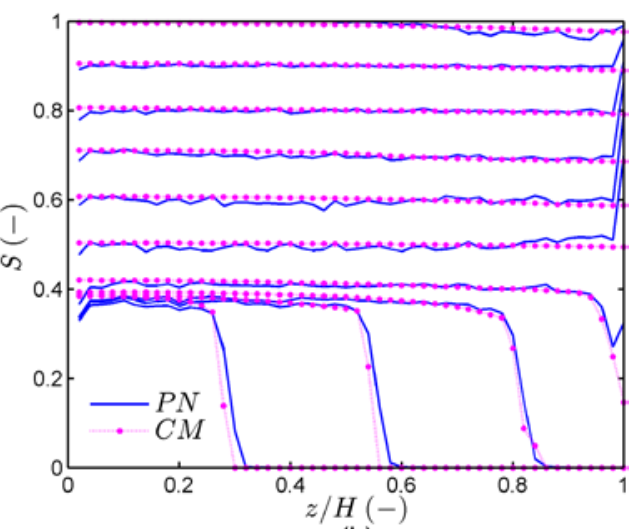

(b)

Figure 6 The continuum model predictions and the pore network simulations: (a) monomodal PSD and (b) bimodal PSD. The saturation profiles of PN are averaged over 10 realizations. From top the profiles belong to network saturation of $0.99,0.9,0.8,0.7,0.6,0.5,0.4,0.3,0.2$, and 0.1 , respectively. 


\section{Conclusions}

In this paper, two different pore network structures (with monomodal and bimodal PSDs) are constructed. The values of moisture transport coefficient in the networks with bimodal PSD are large and confined into a narrow range of saturation in the first drying period. During the receding period of drying, the viscous drag forces dominate the capillary forces in both models. In this period, moisture transport mainly occurs in the vapor phase. Therefore, the moisture transport coefficient profiles are similar in the both pore structures during this drying period. The internal resistance for moisture transport is induced by the formation of liquid clusters and single menisci. The non-local equilibrium effect is observed for the both pore structures and it is more pronounced in the networks with monomodal PSD.

The continuum model is solved and assessed for the both pore structures. It has been seen that the one-equation $\mathrm{CM}$ is able to predict the saturation profiles obtained from the pore networks with monomodal PSD, except those profiles at high local saturation. This is because a small scatter of the moisture transport coefficient value can significantly impact the solution of the CM. The possible reasons for the scattered values could be due to both small network size and averaging method. Nevertheless, the agreement between the CM solutions and the PN simulations is improved when the networks with bimodal PSD are used.

\section{Acknowledgments}

This work was financed by the German Research Foundation (DFG) within the Graduate School 1554 "Micro-Macro-Interactions in Structured Media and Particulate Systems”.

\section{References}

[1] S. Whitaker, Simultaneous heat, mass, and momentum transfer in porous media: A theory of drying, in: J.P. Hartnett, T.F. Irvine (Eds.), Advances in Heat Transfer, Elsevier1977, pp. 119-203.

[2] A.G. Yiotis, A.K. Stubos, A.G. Boudouvis, Y.C. Yortsos, A 2-D pore-network model of the drying of single-component liquids in porous media, Advances in Water Resources 24 (2001) 439-460.

[3] M. Prat, Recent advances in pore-scale models for drying of porous media, Chemical Engineering Journal 86 (2002) 153-164.

[4] T. Metzger, A. Irawan, E. Tsotsas, Isothermal drying of pore networks: Influence of friction for different pore structures, Drying Technology 25 (2007) 49-57.

[5] A.A. Moghaddam, M. Prat, E. Tsotsas, A. Kharaghani, Evaporation in capillary porous media at the perfect piston-like invasion limit: Evidence of nonlocal equilibrium effects, Water Resources Research 53 (2017) 10433-10449. 